\title{
BMJ Open B Part of It School Leaver protocol: an observational repeat cross-sectional study to assess the impact of a meningococcal serogroup B (4CMenB) vaccine programme on carriage of Neisseria meningitidis
}

\author{
Helen S Marshall, ${ }^{\oplus 1,2}$ Mark McMillan, ${ }^{\oplus 1,2}$ Ann Koehler, ${ }^{3}$ Andrew Lawrence, ${ }^{4}$ \\ Jenny MacLennan, ${ }^{5}$ Martin Maiden, ${ }^{5}$ Mary Ramsay, ${ }^{6}$ Shamez N Ladhani, ${ }^{6}$ \\ Caroline Trotter, ${ }^{6,7}$ Ray Borrow, ${ }^{8}$ Adam Finn, ${ }^{9}$ Thomas Sullivan, ${ }^{10}$ Peter Richmond, ${ }^{11}$ \\ Charlene Kahler, ${ }^{12}$ Jane Whelan, ${ }^{13}$ Kumaran Vadivelu ${ }^{14}$
}

To cite: Marshall HS, McMillan M, Koehler A, et al. B Part of It School Leaver protocol: an observational repeat cross-sectional study to assess the impact of a meningococcal serogroup $B$ (4CMenB) vaccine programme on carriage of Neisseria meningitidis. BMJ Open 2019;9:e027233. doi:10.1136/ bmjopen-2018-027233

- Prepublication history and additional material for this paper are available online. To view these files, please visit the journal online (http://dx.doi org/10.1136/bmjopen-2018027233).

Received 17 October 2018 Revised 12 February 2019 Accepted 6 March 2019

Check for updates

(c) Author(s) (or their employer(s)) 2019. Re-use permitted under CC BY-NC. No commercial re-use. See rights and permissions. Published by BMJ.

For numbered affiliations see end of article.

Correspondence to

Professor Helen S Marshall;

helen.marshall@adelaide.edu.au

\section{ABSTRACT}

Introduction Invasive meningococcal disease is uncommon but associated with a high-case fatality rate. Carriage prevalence of the causative bacteria, Neisseria meningitidis, is high in adolescents. A large $(n=34500)$ cluster randomised controlled trial (RCT) to assess the impact of a meningococcal $B$ (MenB) vaccine on meningococcal carriage was implemented in the state of South Australia (SA) for year 10,11 and 12 senior school students in 2017-2018. This study will assess the impact of MenB vaccine (4CMenB) on carriage prevalence in school leavers in SA, 1 and 2 years after implementation of the cluster RCT in adolescents. Measuring the impact of population programmes on carriage can assist in informing future meningococcal immunisation programmes such as targeted age groups and use of catch-up campaigns.

Methods and analysis This repeat cross-sectional study will assess carriage prevalence in 2018 and 2019. All school leavers who attended year 12 in any school in SA in 2018 or 2019 will be invited to participate in this study. An oropharyngeal swab will be taken from each participating student and a risk factor questionnaire completed by the student following informed consent. Students will attend clinics at SA universities, technical colleges, and metropolitan, rural and remote government council clinics. Confirmed vaccination history will allow a comparison in carriage prevalence between vaccinated and unvaccinated school leavers. A sample size of 4096 students per year will provide $80 \%$ power to detect a $20 \%$ difference in carriage prevalence of disease-causing meningococci (defined as genogroup A, B, C, W, X or Y) between years. Ethics and dissemination The study was approved by the Women's and Children's Health Network Human Research Ethics Committee. Results will be published in international peer review journals and presented at national and international conferences.

Trial registration number NCT03419533; Pre-results
Strengths and limitations of this study

This repeat cross-sectional study to assess carriage prevalence in adolescents 15-25 years of age who have completed school will provide the opportunity to assess carriage rates at the population level following introduction of a meningococcal $B$ vaccine programme in younger adolescents who were vaccinated in a school programme (15-18 years of age).

- This study will be one of a limited number of studies that has confirmed participant vaccination history, and that will allow an accurate comparison in carriage prevalence between vaccinated and unvaccinated school leavers.

- Risk factors associated with oropharyngeal carriage will be adjusted for in the analysis; however, potential bias is inherent in observational cross-sectional study designs.

\section{INTRODUCTION}

Neisseria meningitidis (meningococcus) is one of the most common causative organisms of bacterial meningitis in children and young adults $^{1}$ and is associated with a substantial burden of disease including long-term disability, with a case fatality rate of $5 \%-10 \%$. $N$. meningitidis infection also causes significant morbidity and mortality worldwide with approximately 500000-1 200000 cases and $50000-135000$ deaths reported annually. ${ }^{23}$

$N$. meningitidis is classified into serogroups according to the biochemical composition of the capsular polysaccharide. ${ }^{24}$ The serogroup can be predicted by the detection of capsular biosynthesis genes which is interpreted as a corresponding genogroup. In this instance, 
genogroup refers to a meningococcus that has the genetics of a particular serogroup but does not necessarily express the capsule polysaccharide. ${ }^{5}$ The global distribution of meningococcal serogroups/genogroups is temporally and geographically dynamic. Clinically, invasive meningococcal disease (IMD) is most associated with isolates expressing serogroup A, B, C, W, X or Y. ${ }^{6}$ Serogroups B and C together account for a large majority of cases in Europe and the Americas, although serogroup $\mathrm{Y}$ emerged as a major contributor in the 1990s in North America ${ }^{67}$ Serogroup A was responsible for the most recent epidemic in the sub-Saharan meningitis belt in 2009 but is not the causative agent of epidemics elsewhere in the world. Following the introduction of MenAfriVac the incidence of serogroup A-related IMD has dramatically reduced but has been replaced by an increasing burden of serogroup W and C IMD. ${ }^{6}$

From 2002 to 2015 the predominant meningococcal serogroup in Australia associated with IMD was serogroup B. ${ }^{8}$ Since 2013, serogroup W (MenW) and more recently serogroup Y (MenY) have emerged as significant causes of IMD although serogroup B remains the predominant serogroup in 2018 to date. ${ }^{9}$ In Australia, the incidence of IMD due to serogroup B is highest in children under 1 year of age, followed by adolescents between the ages of 15 and 24 years. ${ }^{10}$ In South Australia's (SA) population of 1.7 million people, ${ }^{11}$ serogroup B has remained the predominant serogroup since national surveillance commenced in $1994 .^{12}$

In 2017 in SA there were 36 IMD cases (22 due to serogroup $\mathrm{B}, 11$ serogroup $\mathrm{W}$ and 3 serogroup $\mathrm{Y}$ ). Of these 36 cases, 13 were in children aged $<5$ years $(8$ serogroup B, 4 serogroup $\mathrm{W}, 1$ serogroup $\mathrm{Y}$ ), one of whom died (serogroup B); there was 1 case in 5-9year-olds and 1 case in 10-14year-olds (both due to group W), 4 cases in adolescents aged 15-19 years (4 serogroup B), 3 cases in 20-24year-olds (2 serogroup B, 1 serogroup $W$ ) and 14 cases ( 8 serogroup B, 4 serogroup W, 2 serogroup $\mathrm{Y}$ ) and 2 deaths (1 serogroup B, 1 serogroup W) in adults 25-78 years of age (A Koehler, Communicable Disease Control Branch, SA Health, personal communication, 18 September 2018).

In 2017, 380 IMD cases were notified nationally with a notification rate of 1.5/100 000 compared with a notification rate of 2.1/100 $000 \mathrm{in} \mathrm{SA}$. The proportion of IMD cases due to serogroup B is consistently highest in SA (60\% in 2017), compared with $36 \%$ nationally. ${ }^{13}$

In 2017 the national notification rate for children $<5$ years was 5.5/100 000 and 2.8/100 000 in adolescents 15-19 years of age. From 2000 to $2017,{ }^{13}$ notification rates of serogroup B-related IMD in South Australian $15-19$ year-olds ranged from $1.4 / 100000$ in 15 year-olds to a peak of 6.2/100 000 in 19 year-olds. ${ }^{14}$ The 19 years old age group often coincides with increased social and intimate contact involving activities such as finishing school and commencing higher education, employment or travelling.

IMD notification rates for Aboriginal and Torres Strait Islander people were over seven times the rate for non-Aboriginal people (9.3/100 000 compared with $1.3 / 100000$ ) in 2017 , with disease due to serogroup B being four times higher for Aboriginal people (2.0/100 000 compared with $0.5 / 100000$ nationally). ${ }^{8}$

Exposure to $N$. meningitidis is common in the population, usually only leading to asymptomatic pharyngeal carriage. Although all IMD isolates possess a functional capsule synthesis locus, most carriage isolates are defined by the absence of the expression of the capsule, which is an essential virulence determinant for invasive disease. Carriage isolates are frequently acapsulate for one of three reasons: (1) absence of capsule genes (capsule null locus meningococci); (2) downregulation of capsule synthesis during carriage; and (3) mutational inactivation of capsule-encoding genes. ${ }^{15}$

Despite comprehensive population-based surveillance for IMD, data relating to carriage in Australia are scant and very little is known about overall carriage prevalence of $N$. meningitidis, or the proportion of strains that are serogroup B. The only previous published Australian study reported $1.7 \%$ (3 MenY, the remainder were untypeable) carriage prevalence from a sample of 294 school-age children of various ages in Queensland and was conducted in 1989. ${ }^{16}$ A pilot study conducted in 421 South Australian first year university students in 2017 showed an overall carriage rate of $6.2 \%$ and carriage rate of IMD-associated meningococci of $5.3 \%$.

Host age is one of the most important factors influencing carriage, with peak carriage occurring at around 18-19 years of age. ${ }^{17}$ Other factors that influence carriage include male gender, concomitant respiratory infections, active and passive smoking, number and closeness of social contacts and low socioeconomic status. ${ }^{18} 19$ The relationship between carriage and disease is not fully understood. With carriage rates significantly higher in adolescents, a reduction in carriage in this group has the potential to provide protection to unvaccinated people, including infants, through reduced transmission (herd immunity).

Two new vaccines, Bexsero 4CMenB (GSK) and Trumenba MenB-FHbp (Pfizer), which provide protection against meningococcal $\mathrm{B}$ (MenB) disease, are licensed in Australia but not funded through the National Immunisation Program. There is currently limited evidence that $4 \mathrm{CMenB}$ prevents carriage and no evidence for MenBFHbp. In the UK, a multicentre randomised controlled study was conducted to examine carriage in university students aged 18-24years. ${ }^{20}$ Vaccination with $4 \mathrm{CMenB}$ resulted in significantly lower carriage of any meningococcal strain (18.2\%; 95\% CI $3.4 \%$ to $30.8 \%$ ) carriage reduction, and $26.6 \%$ (95\% CI $10.5 \%$ to $39.9 \%$ ) reduction in capsular groups BCWY. A significant carriage reduction for disease-associated sequence types of capsular B meningococci compared with controls was not observed $(12.6 \%$; $95 \% \mathrm{CI}-15.9 \%$ to $34.1 \%) .{ }^{20}$ As these results are inconclusive, we sought to further assess the indirect (herd protection) effects of $4 \mathrm{CMenB}$ on acquisition of carriage by vaccinating senior school students in 2017 
and 2018 as part of a cluster randomised controlled trial (RCT) in SA. ${ }^{21}$ Over $95 \%$ of schools in SA participated in the study and were randomised to vaccine (vaccination in 2017) or control (vaccination in 2018) arms, with approximately $70 \%$ of year $10,62 \%$ of year 11 and $58 \%$ of year 12 students (final school year) from participating schools consenting to the study.

Our proposed cross-sectional study will assess the impact of vaccinating over 30000 senior school students in 2017-2018 on carriage prevalence in school leavers in SA at 1 and 2-year time points after implementation of the cluster randomised trial. ${ }^{21}$ Carriage prevalence in 2018 will be compared with carriage prevalence in 2019 . Overall effect will be estimated, taking account of the outcome for an average individual irrespective of vaccination status rather than just the direct effect that was estimated in the RCT.

\section{METHODS AND ANALYSIS Study design}

This is a repeat cross-sectional study to assess carriage prevalence in school leavers (first year after final year of senior school) in 2018 and 2019 after offering 4CMenB vaccination to senior school students in SA in 2017 and 2018. Risk factors for carriage in school leavers will also be assessed. Individuals eligible to be enrolled into this study are South Australian school leavers between 17 and 25 years of age in 2018 and 2019. There are no exclusion criteria. School leavers who attended year 12 in any school in SA in 2017 will be invited to participate in 2018. Similarly, in 2019, school leavers who attended year 12 in any school in SA the preceding year (2018) will be approached to participate. An oropharyngeal swab will be taken from each participating student and a risk factor questionnaire completed by the student following informed consent. The questionnaire will collect information on potential risk factors (prior meningococcal vaccination, smoking history, household size, recent antibiotic use, use of mouthwash, intimate kissing and socialising in pubs and clubs) for carriage of N. meningitidis (online supplementary file). Vaccination will be confirmed in students who previously participated in the high school cluster randomised trial through the original study database. Participants who did not take part in the cluster randomised trial will be asked to provide consent to contact their immunisation provider to confirm vaccination history. No ongoing visits are required. Participants will be offered a $\$ 40$ voucher to compensate them for travel expenses.

\section{Recruitment of study participants}

School leavers will be invited to enrol in the study by advertising in the universities and technical colleges, and through council immunisation clinics in 2018. Students previously enrolled in the high school cluster randomised trial will be contacted directly and invited to participate in the study. We will recruit students from the three universities in SA: The University of Adelaide, University of South Australia and Flinders University and university colleges. Students will also be recruited from technical colleges and community immunisation clinics in metropolitan, rural and remote areas of SA to ensure all school leavers in SA (n 19000 per year) are approached and have an opportunity to participate.

At the end of each preceding year, a letter will be provided to schools to distribute to all students in their final year of school to invite them to participate. We aim to recruit 4096 school leavers each year from February to June in 2018 and 2019.

\section{Objectives}

\section{Primary objective}

- Estimate the difference in carriage prevalence of disease-causing genogroups of $N$. meningitidis (A, B, C, W, X, Y) between South Australian school leavers in 2018 (year 12 in 2017) and 2019 (year 12 in 2018), following implementation of a school immunisation programme (4CMenB vaccine) in year 10, 11 and 12 South Australian students from 2017 to 2018.

\section{Secondary objectives}

- Estimate the difference in carriage prevalence of all N. meningitidis genogroups between South Australian school leavers in 2018 (year 12 in 2017) and 2019 (year 12 in 2018).

- Estimate the carriage prevalence of each N. meningitidis genogroup (A, B, C, W, X, Y) in South Australian school leavers in 2018 (year 12 in 2017) and 2019 (year 12 in 2018).

- Across years, estimate the difference in carriage prevalence of $N$. meningitidis genogroups causing disease (A, B, C, W, X, Y) between South Australian school leavers who received $4 \mathrm{CMenB}$ vaccine and unvaccinated students.

- Across years, estimate the difference in carriage prevalence of all N. meningitidis genogroups between South Australian school leavers who have received $4 \mathrm{CMenB}$ and unvaccinated students.

- Across years, estimate the carriage prevalence of each N. meningitidis genogroup (A, B, C, W, X, Y) in South Australian school leavers who received $4 \mathrm{CMenB}$ vaccine and unvaccinated students.

- Identify characteristics associated with carriage prevalence of all $N$. meningitidis genogroups in South Australian school leavers in 2018/2019.

- Identify characteristics associated with carriage prevalence of $N$. meningitidis genogroups causing disease (A, B, C, W, X, Y) in South Australian school leavers in $2018 / 2019$.

\section{Exploratory objectives}

- Describe N. meningitidis carriage density for all genogroups in school leavers using qPCR.

- Describe genome sequencing of pathogenic N. meningitidis (, , B, C, W, X, Y) in carriage among school leavers. 


\section{Study processes}

Oropharyngeal swabs will be collected from all students following informed consent. Students will provide their own consent (parental consent is not required for 17-year-old participants). A standard operating procedure will be used to ensure consistent and optimal collection and handling of swabs. Auditing will occur at sites by randomised process and will be independent from the investigators and sponsor.

\section{Specimen preparation, handling and storage}

A $2 \mathrm{~mL}$ skim milk-tryptone-glucose-glycerol (STGG) medium will be used for collection of oropharyngeal swabs for both molecular and classical bacteriological culture methodologies. Once the samples reach the laboratory, details will be entered into the Laboratory Information System for recording, workup and subsequent reporting. All specimens will be subjected to DNA extraction using the Roche MagNa Pure extraction platform for subsequent molecular testing using routine and standard protocols. Following DNA extraction the remaining STGG medium will be stored at $-80^{\circ} \mathrm{C}$. During transport from collection centres to the central laboratory, swabs will be kept cool using validated transport methods for biological specimens. The majority of oropharyngeal swabs will be couriered to the laboratory for testing within 6 hours. All samples are expected to arrive within 16 hours of being taken, to minimise the loss of cultured isolates due to delayed freezing. ${ }^{22}$

\section{Laboratory processes}

All specimens will be subjected to PCR screening for the presence of specific meningococcal DNA (using porA gene detection) prior to being frozen. Further molecular analysis will be used to determine which genogroup has been detected (A, B, C, W, X and Y). Any samples yielding a positive PCR will be cultured for Neisseria sp on selective agar (NIMM, Micro Neisseria Medium [VCTA], BioMerieux Australia), incubated in $\mathrm{CO}_{2}$ at $35^{\circ} \mathrm{C}$. Plates will be examined daily for isolates for up to 72 hours. $N$. meningitidis will be identified by standard diagnostic laboratory bacteriological methods using oxidase reaction and matrix-assisted laser desorption/ionisation time-offlight mass spectrometer. Whole genome sequencing and metagenomics will be conducted on cultured isolates, as described previously. ${ }^{22}$ From this will be determined serogroup, multilocus sequence type, por $A$ and por $B$ type. Quantitative PCR will be applied to the positive screen samples for estimation of the density of carriage of the Neisseria sp. ${ }^{23}$ A standard curve will be generated allowing comparison of crossing point values from the specimen analysis with the standard curve allowing the estimation of Neisseria density in the specimen.

The SA Pathology laboratories are accredited by National Association of Testing Authorities/Royal College of Pathologists of Australasia for routine testing of human and biological samples. All protocols are documented and reviewed regularly.

\section{Data management}

All data (consent forms, questionnaires and swab results) will be securely stored (password protected) on a database held by The University of Adelaide. Range and logic checks will be performed on all data. All data will be deidentified prior to presentation or publication of results. The final data set will be held by Adelaide Health Technology Assessment, The University of Adelaide.

\section{Statistical analysis}

Carriage prevalence of $N$. meningitidis genogroups as measured by PCR in school leavers is the primary endpoint for statistical analysis. Logistic regression with adjustment for confounding will be used to identify factors associated with carriage and to compare carriage rates annually and between vaccinated and unvaccinated students. Confounders will be identified separately for each outcome/comparison. Relationships will be described using ORs and 95\% CIs.

All analyses will be undertaken according to a prespecified statistical analysis plan. All analyses will be performed using SAS V.9.4.

\section{Sample size estimation}

Assuming the carriage prevalence of disease-associated genogroups of $N$. meningitidis $(\mathrm{A}, \mathrm{B}, \mathrm{C}, \mathrm{W}, \mathrm{X}, \mathrm{Y})$ in school leavers in 2018 is $8 \%$, a sample size of 4096 students per year will provide $80 \%$ power to detect a $20 \%$ relative reduction in carriage prevalence between 2018 and 2019 (two-tailed alpha $=0.05){ }^{24}$

\section{Patient and public involvement}

Study materials were reviewed by the Women's and Children's Health Network Youth Advisory Group. A communications officer will work with school leavers including university students, students attending technical colleges and those in the workforce to establish appropriate and accessible avenues of communication. Involving students in the planning and delivery of communication strategies is expected to facilitate two-way communication and provide opportunities for students to engage in research. Meningitis and meningococcal disease charities in Australia (Meningitis Centre Australia) and the UK (Meningitis Research Foundation) have been contacted and are aware of the proposed research and will assist in promoting awareness of the study to the public.

\section{DISCUSSION}

Evidence of indirect protection in addition to direct protection is an important consideration in implementation of meningococcal vaccine programmes. A carriage study of a cohort of school leavers in SA provides a unique opportunity to assess carriage prevalence at the population level following introduction of an intervention in younger age groups (years 10, 11, 12) and compare carriage in individuals who have previously been vaccinated (in years 11 and 12) with individuals who are not 
vaccinated. In SA, adolescents are generally 15 years of age during year 10 at school. This cross-sectional carriage study of school leavers is being undertaken in the age group with the highest rates of meningococcal carriage and invasive disease in SA. We will also assess any association between various known risk factors and history of $4 \mathrm{CMenB}$ vaccine on prevalence of nasopharyngeal carriage. Although MenC vaccines have shown impressive herd immunity effects, the new MenB vaccines are proteinbased vaccines and their impact on carriage and potential to confer herd protection remains inconclusive. ${ }^{20}$

The success of previously introduced meningococcal $\mathrm{C}$ vaccine programmes in the UK and Australia can be attributed to the combined efficacy of the vaccine against disease and carriage. ${ }^{19}$ Herd protection following introduction of the MenC programme in the UK was determined as $67 \%$ after 1 year increasing to $75 \%$ after 2 years. ${ }^{25}$ Experience with conjugate vaccines suggests protection is afforded by prevention of acquisition of carriage and transmission among the unvaccinated population and when infected, by the presence of antibodies at the time of infection. ${ }^{26-28}$

Evidence supporting a similar impact on carriage to that seen with the MenC vaccine programme is lacking, with inconsistent findings, even with other meningococcal conjugate vaccines. A cross-sectional study assessing the impact of MenACWY vaccine on carriage in a cohort of mostly vaccinated university students showed no significant effectiveness against carriage of MenW or MenY strains at least 5 months after vaccination; with continuing expansion of the 2013 strain of MenW continued in this population despite around $70 \%$ MenACWY vaccine coverage. $^{29}$

In another study assessing the impact of $\mathrm{CRM}_{197}$-based conjugate vaccines MenACWY-CRM and 4CMenB on carriage, an impact on carriage was observed with the MenACWY-CRM vaccine on serogroups CWY, with significantly lower carriage rates for serogroups CWY (36.2\% [95\% CI $15.6 \%$ to $51.7 \%$ ] reduction) and, specifically, serogroup Y (39.0\% [95\% CI $17.3 \%$ to $55.0 \%$ ] reduction) when assessed from 2 months after vaccination. ${ }^{20}$ These lower carriage rates are thought to result mostly from reduction in acquisition rather than increased carriage clearance in vaccinated individuals. Although 4CMenB did not result in a significant reduction in serogroup B carriage, there were significant differences in carriage of meningococci expressing genogroups BCWY and CWY, as well as genogroups CWY, capsular group Y and genogroup $Y$ from 3 months after vaccination with two doses. ${ }^{20}$

In healthy carriers, the diversity of genotypes is wide, with hypervirulent complexes carried rarely. Many hypervirulent strains are poor colonisers, including the ST-11 complex, whereas the ST-23 complex is well adapted to a commensal relationship with the host. ${ }^{18}$ Our pilot study of pharyngeal carriage in university students showed no carriage of the hyperinvasive serogroup B strain harbouring the PorA 1.7, 2-4 variant which causes most cases of invasive disease in SA. ${ }^{22}$ It is not currently known what impact the introduction of protein-based, recombinant meningococcal vaccines will have on interstrain competition and carriage of non-disease-causing meningococci. Information on carriage in this group of adolescents, who only received a MenB vaccine, and not meningococcal ACWY vaccines, will also help understand population effects of the MenB vaccine including any potential genotypic or phenotypic changes in genetic diversity or vaccine antigen expression during this period.

Carriage studies are important to improve our understanding of the population aspects of $N$. meningitidis. Measuring the impact of population programmes on carriage can assist in informing future modifications to programmes such as age groups to be targeted and use of catch-up campaigns

\section{ETHICS AND DISSEMINATION}

Results will be published in international peer review journals and presented at national and international conferences. The study findings will be provided in public forums and to study participants, participating schools and through social media. Results will also be reported in the commercial media including television, radio and print media. The protocol, informed consent forms, recruitment materials, social media and all participant materials have been reviewed and approved by the WCHN HREC and updated on ClinicalTrials.gov: NCT03419533.

\section{Author affiliations}

${ }^{1}$ Vaccinology and Immunology Research Trials Unit, Women's and Children's Health Network, North Adelaide, South Australia, Australia

${ }^{2}$ Robinson Research Institute and Adelaide Medical School, University of Adelaide, Adelaide, South Australia, Australia

${ }^{3}$ Communicable Disease Control Branch, SA Health, Adelaide, South Australia, Australia

${ }^{4}$ Microbiology Department, SA Pathology, Adelaide, South Australia, Australia ${ }^{5}$ Department of Zoology, University of Oxford, Oxford, UK

${ }^{6}$ Immunisation Department, Public Health England, London, UK

${ }^{7}$ Department of Veterinary Medicine, University of Cambridge, Bristol, UK

${ }^{8}$ Meningococcal Reference Unit, Public Health England, Manchester, UK

${ }^{9}$ School of Cellular and Molecular Medicine, University of Bristol, Bristol, UK

${ }^{10}$ School of Public Health, The University of Adelaide, Adelaide, South Australia, Australia

${ }^{11}$ Wesfarmers Centre for Vaccines and Infectious Diseases, Telethon Institute for Child Health Research, University of Western Australia, Perth, Western Australia, Australia

${ }^{12}$ Marshall Centre for Infectious Disease Research and Training, School of Biomedical Science, University of Western Australia, Perth, Western Australia, Australia

${ }^{13}$ GlaxoSmithKline Vaccines, Amsterdam, Netherlands

${ }^{14}$ GlaxoSmith Kline Vaccines, Rockville, Maryland, USA

Acknowledgements HSM acknowledges support from the National Health and Medical Research Council of Australia: Senior Research Fellow Fellowship (1084951). B Part of It Study team: Su-san Lee, Philippa Rokkas, Kathryn Riley, Christine Heath, Mary Walker, Bing Wang, Michelle Clarke, Sara Almond, Maureen Watson, Melissa Cocca, Louise Goodchild, Lesley McCauley. University of Adelaide: Sarah Scott, Lynette Kelly, Roberta Parshotam, Jamie Dunnicliff, Frances Doyle. Adelaide Health Technology Assessment team: Emma Knight, Andrew Holton, Primali de Silva, Mark Armstrong, Tristan Stark, Scott Wilkinson. SA Pathology: Luke Walters, Mark Turra, Daryn Whybrow. Council immunisation providers: Berri Barmera Council, Booleroo Medical Centre, Broughton Clinic, City of Charles Sturt, Coorong District Council, Country Health SA Local Health Network, Eastern 
Health Authority, Health and Immunisation Management Services, Kadina Medical Associates, District Council of Karoonda East Murray, District Council of Lower Eyre Peninsula, District Council of Loxton Waikerie, Mallee Medical Practices, Mid Murray Council, City of Mitcham, Mount Barker District Council, Nganampa Health Council, City of Onkaparinga, District Council of Peterborough, City of Playford, Pop Up Medics, City of Port Lincoln, Renmark Paringa Council, Royal Flying Doctors Service, Streaky Bay Medical Clinic, Tatiara District Council, City of Tea Tree Gully, District Council of Tumby Bay, Wakefield Plains Medical Clinic, City of West Torrens, Whyalla City Council, Watto Purrunna Aboriginal Primary Health Care Service, Wudinna District Council, District Council of Yankalilla. Reference Group: Don Roberton, Ann Koehler, Maureen Watson, Noel Lally, Paddy Philips, Monica Conway, Carolyn Grantskalns, Ann-Marie Hayes, Naomi Dwyer, Andrew Lawrence, Amo Fioravanti, Lyn Olsen, Alistair Burt, Sarah Robertson, Steve Wesselingh, David Johnson, Debra Petrys, Larissa Biggs, Tahlia Riessen.

Contributors HSM wrote the first draft with assistance from MMcM. AK, AL, JMacL, MM, MR, SNL, CT, RB, AF, TS, PR, CK, JW and KV contributed to the manuscript and all authors approved the final version for publication.

Funding Funding for this study was provided by GlaxoSmithKline Biologicals.

Disclaimer GlaxoSmithKline Biologicals has provided the opportunity to review a preliminary version of this manuscript for factual accuracy but the authors are solely responsible for final content and interpretation. The protocol has undergone review by GlaxoSmithKline's Clinical Research and Development Board (CRDB). The funder is independent of study management and analysis of the data. The authors received no financial support or other form of compensation related to the development of the manuscript.

Competing interests HSM is supported by an NHMRC CDF APP1084951 and is a member of the Australian Technical Advisory Group on Immunisation, Australian Government. HSM is an investigator on vaccine trials sponsored by Industry (GSK, Novavax, Pfizer). HSM's and MMcM's institution receives funding for investigatorled studies from Industry (Pfizer, GSK). HSM and MMcM receive no personal payments from Industry. CT has received a consulting payment from GSK and an honorarium from Sanofi Pasteur. RB performs contract research on behalf of Public Health England for GSK, Pfizer and Sanofi Pasteur. PR is an investigator on vaccine trials sponsored by Industry (GSK, Novavax, Pfizer). PR's institution receives funding for investigator-led studies from Industry (Pfizer, GSK, CSL). PR has been a member of scientific vaccine advisory boards for Industry (Pfizer, GSK, Sanofi) but has not received any personal payments from Industry. AF's institution is in receipt of research funding from GlaxoSmithKline, Pfizer and consultancy fees from Alios BioPharma/Johnson \& Johnson, BioNet-Asia and VBI Vaccines. AF is a member of the UK Department of Health's Joint Committee on Vaccination, chair of the WHO European Technical Advisory Group of Experts and president of the European Society for Paediatric Infectious Diseases, which receives sponsorship for its annual meeting from vaccine manufacturers. KV and JW are employees of the GSK group of companies and hold shares in the GSK group of companies as part of their employee remuneration.

\section{Patient consent for publication Not required.}

Ethics approval Women's and Children's Health Network Human Research Ethics Committee (WCHN HREC).

Provenance and peer review Not commissioned; externally peer reviewed.

Open access This is an open access article distributed in accordance with the Creative Commons Attribution Non Commercial (CC BY-NC 4.0) license, which permits others to distribute, remix, adapt, build upon this work non-commercially, and license their derivative works on different terms, provided the original work is properly cited, appropriate credit is given, any changes made indicated, and the use is non-commercial. See: http://creativecommons.org/licenses/by-nc/4.0/.

\section{REFERENCES}

1. Oordt-Speets AM, Bolijn R, van Hoorn RC, et al. Global etiology of bacterial meningitis: A systematic review and meta-analysis. PLoS One 2018;13:e0198772

2. Chang Q, Tzeng YL, Stephens DS. Meningococcal disease: changes in epidemiology and prevention. Clin Epidemiol 2012;4:237-45.

3. Jafri RZ, Ali A, Messonnier NE, et al. Global epidemiology of invasive meningococcal disease. Popul Health Metr 2013;11:17.

4. Rouphael NG, Stephens DS. Neisseria meningitidis: biology, microbiology, and epidemiology. Methods Mol Biol 2012;799:1-20.

5. Caugant DA. Genetics and evolution of Neisseria meningitidis: importance for the epidemiology of meningococcal disease. Infect Genet Evol 2008;8:558-65.
6. Borrow R, Alarcón P, Carlos J, et al. The Global Meningococcal Initiative: global epidemiology, the impact of vaccines on meningococcal disease and the importance of herd protection. Expert Rev Vaccines 2017:16:313-28.

7. Harrison LH. Epidemiological profile of meningococcal disease in the United States. Clin Infect Dis 2010;50 Suppl 2(Suppl 2):S37-S44.

8. Invasive meningococcal disease national surveillance reports - 1 January to 31 December 2017, Canberra Australia: Australian government, department of health. 2017. http://www.health.gov.au/ internet/main/publishing.nsf/Content/ohp-meningococcal-W.htm (Accessed 14 Dec 2018).

9. Invasive meningococcal disease national surveillance report with a focus on menw - 31 march 2018: Australian government department of health. 2018 http://www.health.gov.au/internet/main/ publishing.nsf/Content/ohp-meningococcal-W.htm (Accessed 1 Sep 2018).

10. Lahra MM, Enriquez RP. Australian meningococcal surveillance programme annual report, 2014. Commun Dis Intell Q Rep 2016;40:E221-8.

11. Australian Bureau of Statistics. Population projections, Australia, 2017 (base) - 2066, Canberra, Australia. 2018. http://www. abs.gov.au/ausstats/abs@.nsf/Latestproducts/3222.0Main\% 20Features92017\%20(base)\%20-\%202066?opendocument\& tabname $=$ Summary $\&$ prodno $=3222$.0\&issue $=2017 \% 20$ (base) $\% 20-\%$ 202066\&num=\&view $=$ (Accessed 13 Dec 2018).

12. Meningococcal - australian meningococcal surveillance programme annual reports, canberra, australia: Australian government department of health. 2016. http://www.health.gov.au/internet/main/ publishing.nsf/content/cda-pubs-annlrpt-menganrep.htm (Accessed 14 Dec 2018).

13. National Notifiable Diseases Surveillance System: Australian Government, Department of Health. 2019 http://www9.health.gov.au/ cda/source/pub menin.cfm (Accessed2 Jan 2019).

14. A meningococcal b vaccine program for south australia: Government of south australia, department of health and wellbeing 2017. http://www.sahealth.sa.gov.au/wps/wcm/connect/public+ content/sa+health+internet/about+us/reviews+and+consultation/ meningococcal+b+program+for+south+australia/meningococcal+b+ program+for+south+australia+-+2018 (Accessed 1 Sep 2018).

15. Oldfield NJ, Green LR, Parkhill J, et al. Limited Impact of Adolescent Meningococcal ACWY Vaccination on Neisseria meningitidis Serogroup W Carriage in University Students. J Infect Dis 2018;217:608-16.

16. Ingram SB, Wilson BJ, Kemp RJ, et al. Neisseria meningitidis in a school population in Queensland. Med J Aust 1990;152:332.

17. Christensen $\mathrm{H}$, May M, Bowen L, et al. Meningococcal carriage by age: a systematic review and meta-analysis. Lancet Infect Dis 2010;10:853-61.

18. Caugant DA, Tzanakaki G, Kriz P. Lessons from meningococcal carriage studies. FEMS Microbiol Rev 2007;31:52-63.

19. MacLennan J, Kafatos G, Neal K, et al. Social behavior and meningococcal carriage in British teenagers. Emerg Infect Dis 2006;12:950-7.

20. Read RC, Baxter D, Chadwick DR, et al. Effect of a quadrivalent meningococcal ACWY glycoconjugate or a serogroup $B$ meningococcal vaccine on meningococcal carriage: an observerblind, phase 3 randomised clinical trial. Lancet 2014;384:2123-31.

21. Marshall HS, McMillan M, Koehler A, et al. B Part of It protocol: a cluster randomised controlled trial to assess the impact of $4 \mathrm{CMenB}$ vaccine on pharyngeal carriage of Neisseria meningitidis in adolescents. BMJ Open 2018;8:e020988.

22. McMillan M, Walters L, Turra M, et al. B Part of It study: a longitudinal study to assess carriage of Neisseria meningitidis in first year university students in South Australia. Hum Vaccin Immunother 2018 (Published Online First: 04 Jan 2019).

23. Finn A, Morales-Aza B, Sikora P, et al. Density distribution of pharyngeal carriage of meningococcus in healthy young adults: New approaches to studying the epidemiology of colonization and vaccine indirect effects. Pediatr Infect Dis J 2016;35:1080-5.

24. Fitzpatrick PE, Salmon RL, Hunter PR, et al. Risk factors for carriage of Neisseria meningitidis during an outbreak in Wales. Emerg Infect Dis 2000;6:65-9.

25. Maiden MC, Ibarz-Pavón AB, Urwin R, et al. Impact of meningococcal serogroup $C$ conjugate vaccines on carriage and herd immunity. J Infect Dis 2008;197:737-43.

26. Dagan R, Givon-Lavi N, Zamir O, et al. Reduction of nasopharyngeal carriage of Streptococcus pneumoniae after administration of a 9 -valent pneumococcal conjugate vaccine to toddlers attending day care centers. J Infect Dis 2002;185:927-36.

27. Dagan R, Melamed R, Muallem M, et al. Reduction of nasopharyngeal carriage of pneumococci during the second year of 
life by a heptavalent conjugate pneumococcal vaccine. $J$ Infect Dis 1996:174:1271-8.

28. Dagan R, Muallem M, Melamed R, et al. Reduction of pneumococcal nasopharyngeal carriage in early infancy after immunization with tetravalent pneumococcal vaccines conjugated to either tetanus toxoid or diphtheria toxoid. Pediatr Infect Dis $J$ 1997:16:1060-4.

29. Oldfield NJ, Cayrou C, AlJannat MAK, et al. Rise in Group W Meningococcal Carriage in University Students, United Kingdom. Emerg Infect Dis 2017;23:1009-11. 